\section{Tattoos and sarcoidosis}

I read, with keen interest, the $C M A J$ article "Tattoo reactions as a sign of sarcoidosis," which described granulomatous tattoo reaction in a patient with systemic sarcoidosis. ${ }^{1}$ Tattoo sarcoidosis has been known for more than half a century, although only a few cases have been described. One hypothesis is that this may be a "pigment-induced disease."' Tattoo pigments may provide chronic antigenic stimulation leading to Th1/Th2 imbalance in a genetically predisposed individual, thus triggering the development of sarcoidosis. This is based on the demonstration of pigment in the tattoo granuloma as well as similar pigment in the noncutaneous site of granulomatous inflammation. On histopathologic examination, pigment granules, pigment laden macrophages or polarizing material may be demonstrable. I am interested in knowing if these findings were seen in any of the histopathologic sections from the patient described by Post and Hull, and when the patient was tattooed. ${ }^{1}$ In 2005 , Antonovich and Callen reviewed the published cases of tattoo sarcoidosis. ${ }^{3}$ Most cases occurred along with pulmonary involvement and tattoo reactions preceded the diagnosis of sarcoidosis in 14 out of 19 cases. The authors also highlighted that granulomatous inflammation on histopathology with demonstrable foreign material should not be used as a criterion to exclude a diagnosis of sarcoidosis, as was the previous school of thought.

\section{Karan Madan MBBS MD DM}

Senior resident, Department of Pulmonary Medicine, Postgraduate Institute of Medical Education and Research, Chandigarh, India

\section{References}

1. Post J, Hull P. Tattoo reactions as a sign of sarcoidosis. CMAJ 2012;184:432.

2. Pandhi D, Sonthalia S, Singal A. Mortimer's Malady revisited: a case of polymorphic cutaneous and systemic sarcoidosis. Indian J Dermatol Venereol Leprol 2010;76:448.

3. Antonovich DD, Callen JP. Development of sarcoidosis in cosmetic tattoos. Arch Dermatol 2005; 141:869-72.

CMAJ 2012. DOI:10.1503/cmaj.112-2029

\section{The authors respond}

We are pleased that our article ${ }^{1}$ was read with keen interest. In response to Madan's letter, ${ }^{2}$ our patient's tattoos were amateur tattoos acquired over many years via a variety of methods, and presumably pigmentary substances were used, although all tattoos were black in colour. While sarcoidal reactions to a particular dye is well known, simultaneous reactions occurring in all the tattoos as well as in the eyes and lungs is more unusual. When a reaction occurs in a tattoo, pigment in the tissue is expected. Determining whether pigment is present in eyes or lungs is more difficult. If the sarcoidal reaction is immune-based, one would expect that a single pigment is responsible. Perhaps more likely is that, as is the case in sarcoidosis occurring in scars, tattoos act to localize the granulomatous reaction as a Koebner phenomenon.

\section{Joel Post JD MD}

Department of Ophthalmology

Peter Hull MB BCh PhD

Division of Dermatology, Department of Medicine, University of Saskatchewan, Saskatoon, Sask.

\section{References}

1. Post J, Hull P. Tattoo reactions as a sign of sarcoidosis. CMAJ 2012;184:432.

2. Madan K. Tattoos and sarcoidosis [letter]. CMAJ 2012;184:563.

CMAJ 2012. DOI:10.1503/cmaj.112-2030

\section{Medication regimens}

In a CMAJ news article, ${ }^{1}$ Roger Collier discussed the benefit of fixed-dose combination drugs. This innovation promotes adherence and is a promising locus for pharmaceutical development, but such pills offer only a partial solution to the challenges associated with multiple-medication treatment regimens.

A 2008 review suggests that high dosing frequency of medications may have the most consistent negative effect on adherence. ${ }^{2}$ In addition to reducing the pill burden, fixed-dose combination drugs may improve adherence through reducing both the frequency of taking medications and the challenges in interpreting how medications are to be taken. These outcomes can also be accomplished when health professionals consolidate instructions on how to take medications into a list that groups medications that can be taken together. Unfortunately, few older patients, who struggle with the most complex medication regimens, will adequately consolidate instructions on their own. ${ }^{3}$

Medication regimen complexity can be evaluated only after consideration is given to how the instructions for taking individual medications fit together when seen as part of a unified script. The $C M A J$ news article ${ }^{1}$ cited justified concern over the lack of customization offered by combination pill approaches. A truly person-centred approach to simplifying medication regimens requires an assessment of regimen complexity in each unique case, including ways to reduce the number of doses and medications. ${ }^{4}$ The individual benefits of therapeutics must be weighed against the costs of multiple-medication regimens when drugs are prescribed concurrently. There are important implications for adherence, patient safety and quality of life.

The simplest medication regimen that meets the mutual and explicitly defined goals of patient and physician is surely the best one. Simplification must go beyond reducing the pill burden through combination pills.

\section{Jonathan Fuller BMSc}

$\mathrm{MD} / \mathrm{PhD}$ student, Faculty of Medicine

\section{Shawn Tracy BSc}

$\mathrm{PhD}$ candidate, Institute of Medical

Science, University of Toronto

Ross E.G. Upshur MD MSc

Professor, Department of Family and

Community Medicine, Sunnybrook Health

Sciences Centre, Toronto, Ont.

\section{References}

1. Collier R. Reducing the "pill burden." CMAJ 2012; 184:E117-8.

2. Ingersoll KS, Cohen J. The impact of medication regimen factors on adherence to chronic treatment: a review of literature. J Behav Med 2008;31:213-24.

3. Wolf MS, Curtis LM, Waite K, et al. Helping patients simplify and safely use complex prescription regimens. Arch Intern Med 2011;171:300-5.

4. Fuller J, Upshur RE. Medication regimen complexity and the care of the chronically ill patient. Int $J$ Person Centered Med 2011;1:719-25.

CMAJ 2012. DOI:10.1503/cmaj.112-2031 